\title{
Retinopatia de Purtscher-like e pancreatite aguda
}

\section{Purtscher-like retinopathy and acute pancreatitis}

Kelly Fernandes de Paula Rodrigues', Carlos Alexandre de Amorim Garcia², Veridiana Puppio Querido', Paulo de Souza Segundo ${ }^{3}$

\section{Resumo}

Retinopatia de Purtscher-like é uma baixa súbita da visão associada à imagem de múltiplas áreas branco-amareladas (manchas algodonosas) e hemorragias no pólo posterior de ambos os olhos. O exato mecanismo da injúria ainda não é claro, mas provavelmente seria de natureza embólica. Tem sido descrita em uma variedade de condições, incluindo pancreatite aguda, síndrome de embolia gordurosa, insuficiência renal, nascimento (parto e pós-parto), desordens do tecido conectivo, entre outras. Serão relatados três casos de pancreatite aguda confirmada pelos exames laboratoriais e história clínica, associadas a alterações no exame do fundo de olho, compatíveis com esta retinopatia.

Descritores: Pancreatite alcoólica/complicações; Pancreatite/complicações; Doenças retinianas/etiologia; Transtornos da visão; Relatos de casos [Tipo de publicação]

\footnotetext{
${ }_{2}^{1}$ Residente em Oftalmologia da Universidade Federal do Rio Grande do Norte - UFRN - Natal (RN), Brasil;

${ }^{2}$ Professor Adjunto do Departamento de Oftalmologia da Universidade Federal do Rio Grande do Norte - UFRN - Natal (RN), Brasil;

${ }^{3}$ Fellow Setor de Retina do Hospital Universitário Onofre Lopes da Universidade Federal do Rio Grande do Norte - UFRN - Natal (RN), Brasil.

Trabalho realizado na Universidade Federal do Rio Grande do Norte - UFRN - Natal (RN), Brasil.
} 


\section{INTRODUÇÃO}

A retinopatia de Purtscher foi descrita pela primeira vez em 1910, sendo identificada por perda visual brusca associada à trauma craniano ${ }^{(1)}$. Esta retinopatia é caracterizada por manchas algodonosas, edema de retina e mácula associados a hemorragias superficiais. Outras condições associadas com retinopatia de Purtscher tem sido descritas, incluindo pancreatite aguda, doenças linfo-proliferativas, transplante de medula óssea, transplante renal, embolia gordurosa, trauma por air bag, manobra de valsalva, pósparto e adenocarcinoma de pâncreas ${ }^{(2-6)}$.

$\mathrm{O}$ primeiro caso relacionado à pancreatite aguda foi publicado em 1975 por Inkeles et al ${ }^{(7)}$. Retinopatia Purtscher-like é caracterizada por súbita redução da acuidade visual associada a sinais oftalmológicos semelhantes a retinopatia de Purtscher $^{(8)}$.

\section{Descrição do Caso Clínico 1:}

Paciente do sexo masculino, 24 anos, solteiro, soldador, com baixa súbita da visão de ambos os olhos iniciada um dia após quadro de forte dor abdominal superior, em faixa constante, que se agravava ao longo do tempo, associada a episódios de vômitos. Relatou ter feito consumo de grande quantidade de bebida alcoólica durante os cinco dias que antecederam o quadro clínico.

Os exames complementares apresentaram: amilase de $1331 \mathrm{U} / \mathrm{dl}$ e hemograma sem anormalidade. USG abdominal total com presença de líquido livre na cavidade peritonial e diagnóstico sugestivo de pancreatite aguda. Tomografia computadorizada demonstrou pancreatite aguda edematosa com fleimão pancreático e presença de área de necrose em corpo e cauda (Baltazar classe C).

Após sete dias do episódio inicial, encaminhado ao setor de oftalmologia do Hospital Universitário Onofre Lopes, apresentava acuidade visual no olho direito de movimento de mão e no esquerdo de conta dedos a meio metro. Biomicroscopia sem anormalidades e no fundo de olho, de ambos os olhos, presença de numerosas manchas algodonosas dispostas, principalmente no pólo posterior, circundando os vasos retinianos e microhemorragias (Figura 1).

A angiografia fluoresceínica mostrou áreas de não perfusão capilar e pontos de hipofluorescência por bloqueio, correspondentes as hemorragias (Figura 2).

Após dois meses do episódio inicial, clinicamente recuperado da pancreatite, apresentava acuidade de 20/ 40 em ambos os olhos (Figura 3) e perimetria computadorizada com diminuição difusa da sensibilidade em ambos os olhos.

\section{Descrição do Caso Clínico 2:}

Paciente, 18 anos, na 22,3 semanas de gestação, com quadro clínico de dor abdominal em faixa, náuseas e vômitos, apresentou quatro dias após o inicio dos sintomas baixa visual em ambos os olhos. Foi submetida à cirurgia cesariana de urgência e encaminhada ao nosso serviço para avaliação. Ao exame apresentava acuidade visual de 20/40 no (OD) e conta dedos a 20 centímetros no (OE). Biomicroscopia sem anormalidades, pupilas isocóricas, reflexo fotomotor e consensual preservados. Pressão intra-ocular de $10 \mathrm{mmHg}$ em ambos os olhos. Fundo de olho com manchas algodonosas no pólo posterior e edema macular (Figura 4).

Os exames apresentavam amilase 1.900U/dl, transaminase e hemograma normais. Paciente perdeu acompanhamento sendo reavaliada após 8 meses, apresentando na ocasião resolução do quadro oftalmológico sem seqüela na acuidade visual final.

\section{Descrição do Caso Clínico 3:}

Paciente, do sexo masculino, 30 anos, auxiliar de eletrônica, branco, apresentou quadro de dor em região hipogástrica de forte intensidade com vômitos, após ingestão de bebida alcoólica, foi diagnosticado pancreatite aguda no hospital de urgência. Após dois dias do início dos sintomas, apresentou baixa súbita da visão de ambos os olhos, com visão de vultos e melhora progressiva. Passado de cirurgia de pseudocisto no pâncreas há um ano e meio. Apenas após dois meses do episódio procurou o setor de oftalmologia apresentando ao exame acuidade visual de 20/20 - 2 no olho direito e 20/ 60 no olho esquerdo. Biomicroscopia sem anormalidades. PIO de $14 \mathrm{mmHg}$ em ambos os olhos. O exame da retina mostrava exsudatos duros puntiformes esparsos na região macular em ambos os olhos, associado à edema macular no (OE) (Figura 5). Exame de campo visual mostrava escotoma central, principalmente no (OE).

\section{Discussão}

Os casos descritos são de pacientes jovens com diagnóstico de pancreatite aguda, associada à diminuição da acuidade visual e alterações retinianas sugestivas de Purtscher-like. O primeiro e terceiro caso após consumo de grande quantidade de bebida alcoólica. Segundo caso, mulher grávida com gestação interrompida por complicações de pancreatite aguda. O quadro ocular é uma complicação bastante rara de pancreatite aguda e pouco citada na literatura.

A síndrome de Purtscher-like, associada à 


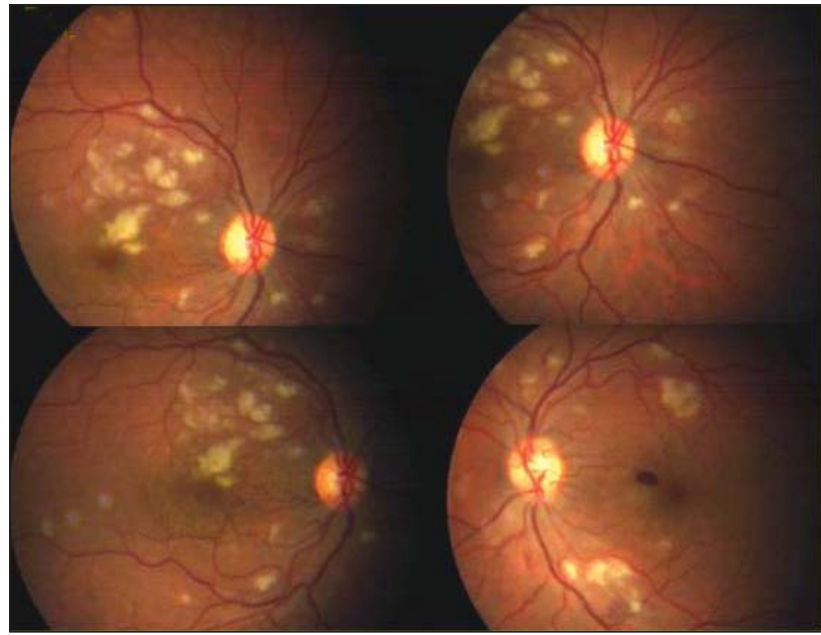

Figura 1: Numerosas manchas algodonosas dispostas, principalmente no pólo posterior e circundando os vasos da retina

pancreatite aguda, é caracterizada pela perda visual, geralmente bilateral, que se desenvolve, mais comumente, nos dois primeiros dias do início da dor abdominal ${ }^{(8-1)}$, associada à presença, na fundoscopia, de exsudatos ou manchas algodonosas dispostas, principalmente no pólo posterior $^{(4,10-11)}$, de forma múltipla, de aspecto superficial, entre as arteríolas e vênulas, podendo também ser encontradas manchas vermelhas pseudocerejas em volta da fóvea, que é comumente vista em oclusão da artéria retiniana central ${ }^{(10)}$, como também presença de microhemorragias ou hemorragias em chama de vela ${ }^{(0,12)}$. Tem sido demonstrado, através de estudos clínicos-patológicos, que a coriocapilar também é afetada nestes casos ${ }^{(8.9)}$. Menos comumente, pode ser relatado ainda descolamento seroso da mácula, hemorragias préretinianas, vasos dilatados e edema do disco óptico ${ }^{(10)}$.

O mecanismo fisiopatológico exato ainda é controverso. É proposto a ocorrência de êmbolo, que levaria à oclusão arteriolar e conseqüente infarto da microvasculatura na camada de fibras nervosas ${ }^{(4,6,8,10)}$. Este êmbolo seria relacionado à liberação, na circulação sistêmica, de enzimas proteolíticas procedentes do pâncreas inflamado ${ }^{(7)}$. A agregação leucocitária seria induzida pela ativação total do complemento C5 para $C 5 a$, levando a ativação da cascata do complemento ${ }^{(4,6,8,10-12)}$. Este é tido como o mecanismo mais aceito por estar associado tanto ao trauma, quanto a pancreatite aguda e as doenças vasculares. Outros êmbolos potenciais associados à síndrome seriam de gordura, de ar, de agregados leucocitários, plaquetas, de fibrina ou de fluido amniótico ${ }^{(3)}$.

Dessa forma, a síndrome também está associada a período pós-parto devido à provável embolização do fluido amniótico, podendo até cursar em alguns casos

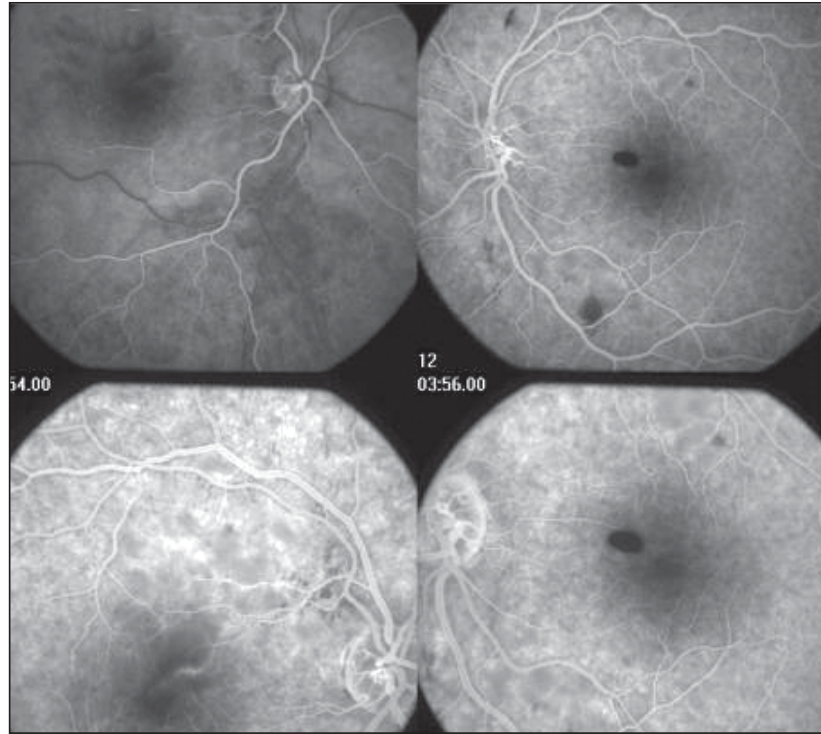

Figura 2: Angiografia fluoresceínica e anerita mostrando áreas de hipofluorescência por bloqueio, não se observando as manchas algodonosas vista na retinografia

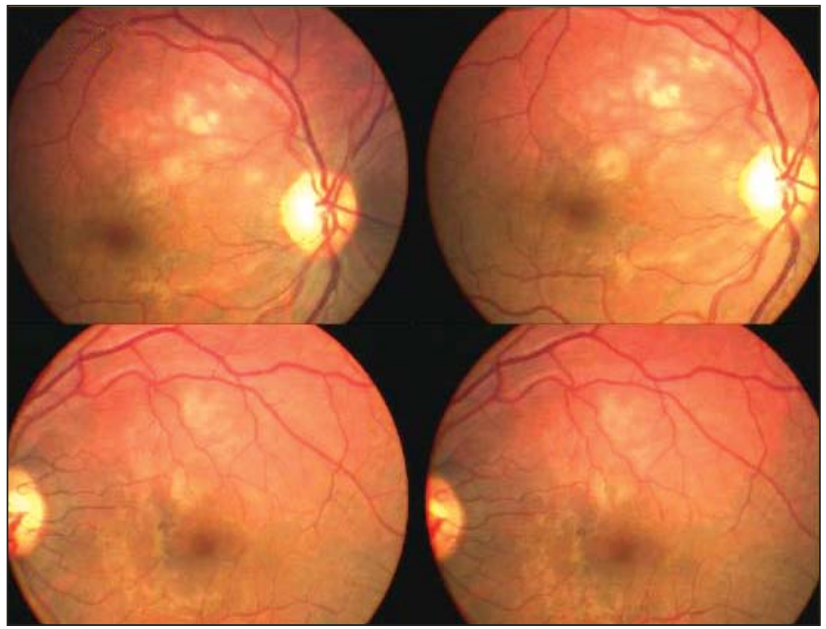

Figura 3: Imagem de fundo de olho, 2 meses após o episódio inicial

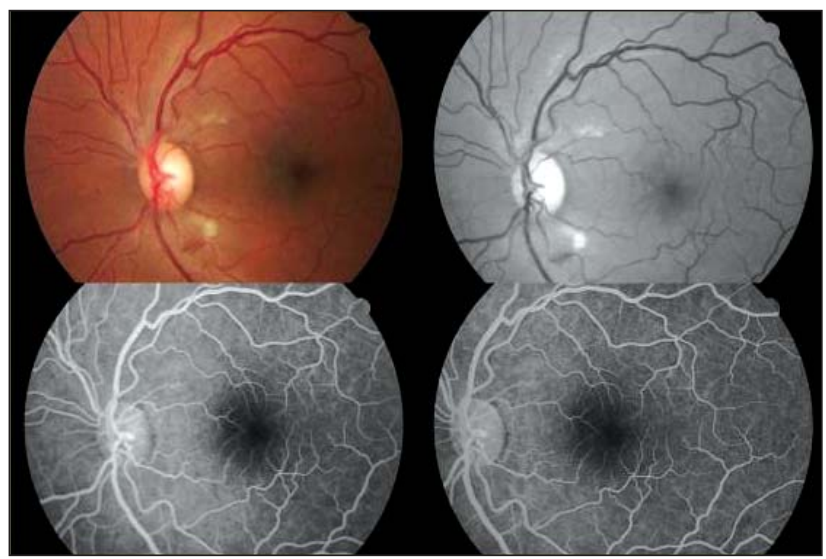

Figura 4: Retinografia e anerita mostrando mancha algodonosa no pólo posterior e angiografia não evidenciando a mesma 


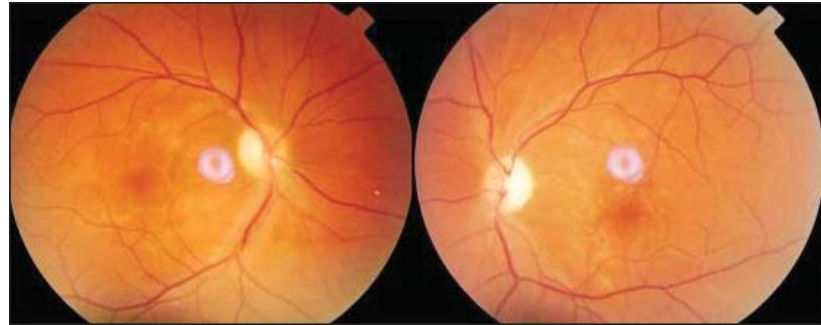

Figura 5: Exame da retina com presença de exsudatos duros, puntiformes e esparsos na região macular, após 2 meses do quadro de pancreatite aguda

com coagulação intravascular disseminada, como préeclâmpsia por infartos intravasculares ${ }^{(10)}$.

A angiografia fluoresceínica pode ajudar na complementação diagnóstica na qual é mostrada isquemia devido à oclusão de pequenas arteríolas retinianas geradas por micropartículas intravas-culares ${ }^{(3)}$. Esta síndrome não tem relação com sexo, raça ou idade, como também não há estatísticas quanto à sua freqüência ${ }^{(10)}$. A recuperação visual é bastante variável e incerta, com resolução das lesões em torno de quatro meses, podendo a função visual se recuperar por completo ou não ${ }^{(8)}$.

Com relação ao tratamento foram mostrados respostas com o uso de esteróides em altas doses, como também descrito o uso da papaverina, mas nada foi definido ${ }^{(11,13)}$. Nestes casos foi realizado apenas o acompanhamento oftalmológico e tratamento clínico da pancreatite.

A síndrome de Purtscher-like é uma complicação ocular rara que pode estar associada à pancreatite aguda, como também a várias desordens sistêmicas. Tem como diagnóstico primordial a história clínica e o exame do paciente ${ }^{(8)}$, acometimento ocular importante, sem tratamento específico definido, com recuperação visual variada e não associada a gravidade da doença de base.

\section{Abstract}

Purtscher-like retinopathy is acute loss of vision associated image of the multiple areas of retinal whitening and hemorrhage in the posterior pole of both eyes. The exact mechanism of injury remains unclear, current evidence suggests that it is embolic in nature. In a variety of conditions are been described including acute pancreatitis, fat embolism syndrome, renal failure, childbirth, and connective tissue disorders. Will are related three cases of the acute pancreatitis which was confirmed by complementary laboratory studies and clinical history, associated from exam of the fundus of the eye, similar is this retinopathy.
Keywords: Pancreatitis alcoholic/complications; Pancreatitis/complications; Retinal diseases/etiology; Vision disorders; Case reports [Publication type]

\section{RefERÊNCiaS}

1. Purtscher O. Angiopathia retinae traumatica; lymphorrhagien des Augengrudes. Graefes Arch Clin Exp. 1912; 82:347-71.

2. Tabandeh H, Rosenfeld PJ,Alexandrakis G, Kronish JP, Chaudhry NA. Purtscher-like retinopathy associated with pancreatic adenocarcinoma. Am J Ophthalmol. 1999; 128(5): 650-2.

3. Carrera CR, Pierre LM, Medina FM, Pierre-Filho Pde T. Purtscher-like retinopathy associated with acute pancreatitis. Sao Paulo Med J. 2005; 123(6):289-91.

4. Sánchez Pedraza R, Párraga Quiles MJ, Barreiro Lucas JC, Gallardo Galera JM, Laborda Oñate JM. Retinopatía de Purtscher asociada a transplante renal. indice Studium [Internet]. 1997; 16(2). Avaliable from URL: http:// www.oftalmo.com/studium/studium1997/stud97-2/b-11.htm

5. Shah GK, Penne R, Grand MG. Purtscher's retinopathy secondary to airbag injury. Retina. 2001; 21(1): 68-9.

6. Stewart MW, Brazis PW, Guier CP, Thota SH, Wilson SD. Purtscher-like retinopathy in a patient with HELLP syndrome. Am J Ophthalmol. 2007; 143(5):886-7.

7. Inkeles DM, Walsh JB. Retinal fat emboli as sequela to acute pancreatitis. Am J Ophthalmol. 1975; 80(5):935-8.

8. López-Tizón E, Reinoso-Montalvo C, Mencía-Gutiérrez E, Gutiérrez-Díaz E. [Acute pancreatitis presenting as sudden blindness]. Arch Soc Esp Oftalmol. 2006; 81(3): 161-3. Spanish.

9. Agrawal A, McKibbin MA. Purtscher's and Purtscher-like retinopathies: a review. Surv Ophthalmol. 2006; 51(2): 12936. Comment in: Surv Ophthalmol. 2007; 52(1):117-8; author reply $118-9$.

10. Chaum E. Retinopathy, Purtscher. In: eMedicine: SpecialitiesOphthalmology- Retina [Internet]. WebMd; c1996-2008. [cited 2007 Nov 12]. Available from: http:// www.emedicine.com/OPH/topic419.htm

11. Safar A, Pillai S. 23 year old man with a history of alcohol abuse. DJO: Digit J Ophthalmol. [Internet]. 1997. [cited 2007 Nov 12]. Avaliable from: http://www.djo.harvard.edu/ site.php?url=/physicians/gr/354

12. Campo SM, Gasparri V, Catarinelli G, Sepe M. Acute pancreatitis with Purtscher's retinopathy: case report and review of the literature. Dig Liver Dis. 2000; 32(8): 729-32.

13. Wang AG, Yen MY, Liu JH. Pathogenesis and neuroprotective treatment in Purtscher's retinopathy. Jpn J Ophthalmol. 1998; 42(4):318-22.

ENDEREÇO PARA CORRESPONDÊNCIA:

Carlos Alexandre de Amorim Garcia

Departamento Oftalmologia - UFRN

Hospital Universitário Onofre Lopes

CEP-59075-250 - Natal - Rio Grande do Norte

E-mail: prontoc.de.olhos@digi.com.br 